\title{
Extensions of No-Go Theorems to Many Signal Systems
}

\author{
Radu Balan
}

\begin{abstract}
In this paper we extend the Balian-Low type theorems to Riesz bases for systems of many signals. We present the construction of coherent frames and we give sufficient conditions for these frames to have coherent duals. Under these conditions we prove some nonlocalization theorems.
\end{abstract}

\section{Introduction}

For two real numbers $a, b$ we introduce on $L^{2}(\mathbf{R})$ two unitary operators:

$$
\begin{gathered}
t_{a, b} f(x)=e^{2 \pi i a x} f(x-b) \\
w(a, b) f(x)=e^{-i \pi a b} e^{2 \pi i a x} f(x-b)
\end{gathered}
$$

for any $f \in L^{2}(\mathbf{R})$. We notice that $w(a, b)=e^{-i \pi a b} t_{a, b}$ and the adjoints are $t_{a, b}^{*}=e^{-2 \pi i a b} t_{-a,-b}, w(a, b)^{*}=w(-a,-b)$. Ignoring the toral component, the operator $w(a, b)$ is the Schrödinger representation of the Weyl-Heisenberg group. In the standard Weyl-Heisenberg frame theory (see [Daub90] or [HeWa89]) one starts with a function $g \in L^{2}(\mathbf{R})$ (the window) and two positive numbers $\alpha, \beta>0$ and constructs the set

$$
\mathcal{G}_{g ; \alpha, \beta}=\left\{t_{m \alpha, n \beta} g ;(m, n) \in \mathbf{Z}^{2}\right\}
$$

obtained by translating and modulating $g$ with parameters from the discrete lattice $\left\{(m \alpha, n \beta) ;(m, n) \in \mathbf{Z}^{2}\right\} \subset \mathbf{R}^{2}$. On the other hand one can proceed in the same way but using $w(a, b)$ instead of $t_{a, b}$. In this case the following set is constructed:

$$
\mathcal{W}_{g, \alpha, \beta}=\left\{w(m \alpha, n \beta) g ;(m, n) \in \mathbf{Z}^{2}\right\}
$$

similar to $\mathcal{G}_{g ; \alpha, \beta}$ except for an extra phase factor in each function. To distinguish between these two sets, we shall call $\mathcal{G}_{g ; \alpha, \beta}$ a Gabor set whereas $\mathcal{W}_{g ; \alpha, \beta}$ will be called a Weyl-Heisenberg set.

1991 Mathematics Subject Classification. Primary 42A99, 43A99; Secondary 94 A11.

Key words and phrases. Gabor Weyl-Heisenberg frames, Balian-Low theorem, timefrequency localization.

The author wants to thank Professor Ingrid Daubechies for the continuous help and support she provided. He also wants to thank the anonymous referee for the helpful comments and suggestions that he made. 
We now recall some definitions and constructions from the frame theory. Consider a (complex) Hilbert space $K$, a countable index set $\mathbf{I}$ and a set $\mathcal{F}=\left\{f_{i}, i \in\right.$ I\} $\subset K$ of elements of $K$. Then:

Definition 1.1. The set $\mathcal{F}$ is called a frame for $K$ if there are two positive constants $0<A \leq B<\infty$ such that for any $x \in K$ :

$$
A\|x\|^{2} \leq \sum_{i \in \mathrm{I}}\left|<x, f_{i}>\right|^{2} \leq B\|x\|^{2}
$$

The positive numbers $A$ and $B$ are called (frame) bounds. If they can be chosen equal (i.e. $A=B$ ) then the frame is called tight.

Definition 1.2. The set $\mathcal{F}$ is called a Riesz basis of $K$ if it is frame for $K$ and it is also a Schauder basis.

For a frame $\mathcal{F}$ we introduce the following bounded operator, called the analysis operator:

$$
T: K \rightarrow l^{2}(\mathbf{I}), T(x)=\left\{<x, f_{i}>\right\}_{i \in \mathbf{I}}
$$

where $l^{2}(\mathbf{I})$ is the space of square summable complex sequences indexed by $\mathbf{I}$. The adjoint of $T$, called the synthesis operator, is given by:

$$
T^{*}: l^{2}(\mathbf{I}) \rightarrow K, T^{*}(c)=\sum_{i \in \mathbf{I}} c_{i} f_{i}
$$

Let us denote by $S=T^{*} T$ the positive operator called the frame operator:

$$
S: K \rightarrow K, S(x)=\sum_{i \in \mathrm{I}}<x, f_{i}>f_{i}
$$

We see that (1.5) is equivalent to the following operatorial inequalities:

$$
A \cdot 1 \leq S \leq B \cdot 1
$$

Using $S$ we introduce two special frames: the standard dual frame, defined by:

$$
\tilde{f}_{i}=S^{-1} f_{i}, i \in \mathbf{I}
$$

and the associated tight frame, defined by:

$$
f_{i}^{\#}=S^{-1 / 2} f_{i}, i \in \mathbf{I}
$$

The standard dual frame $\tilde{\mathcal{F}}=\left\{\tilde{f}_{i}, i \in \mathbf{I}\right\}$ has the following reconstruction property:

$$
x=\sum_{i \in \mathrm{I}}<x, f_{i}>\tilde{f}_{i}=\sum_{i \in \mathrm{I}}<x, \tilde{f}_{i}>f_{i}, \forall x \in K
$$

whereas the associated tight frame $\mathcal{F}^{\#}=\left\{f_{i}^{\#}, i \in \mathbf{I}\right\}$ is a tight frame with frame bound 1 (see [HeWa89]).

Now, returning to Gabor and Weyl-Heisenberg sets, we notice that $\mathcal{G}_{g ; \alpha, \beta}$ is frame if and only if $\mathcal{W}_{g ; \alpha, \beta}$ is frame.

The classical Balian-Low theorem states that if $\mathcal{G}_{g ; \alpha, \beta}$ is an orthonormal basis for $L^{2}(\mathbf{R})$ then $g$ is nonlocalized, i.e. $x \mapsto x g(x)$ and $x \mapsto g^{\prime}(x)$ cannot both be in $L^{2}(\mathbf{R})$ (see references in [Bali81], [Low85]). This result was later extended to the case when $\mathcal{G}_{g ; \alpha, \beta}$ is a Riesz basis for $L^{2}(\mathbf{R})$ (see [Daub90] or [BHW 95]).

Although it appears that the extra phase factor in (1.4) is harmless, we shall see that this is not true for many signals systems. In the case when (1.3) or (1.4) is a frame we shall call it a Gabor frame, respectively a Weyl-Heisenberg frame. In 
this paper we shall use the term coherent as meaning of Gabor or Weyl-Heisenberg type.

Let us denote by $L^{2}\left(\mathbf{R}, \mathbf{C}^{n}\right)=L^{2}(\mathbf{R}) \oplus \ldots \oplus L^{2}(\mathbf{R})$ the direct sum of $k$ copies of $L^{2}(\mathbf{R})$. Our goal is to extend the Balian-Low theorem to frames in $L^{2}\left(\mathbf{R}, \mathbf{C}^{n}\right)$. We point out that our approach is different to the one followed by Zeevi and Zibulski (see [ZiZe95]).

The organization of the paper is the following: in section 2 we describe coherent frames for $L^{2}\left(\mathbf{R}, \mathbf{C}^{n}\right)$ with coherent duals; in section 3 we give the no-go theorems for Riesz bases; section 4 contains the conclusions and is followed by the bibliography.

\section{Construction of coherent frames with coherent duals}

Let us consider the Hilbert space $L^{2}\left(\mathbf{R}, \mathbf{C}^{n}\right)=L^{2}(\mathbf{R}) \oplus \ldots \oplus L^{2}(\mathbf{R})$ endowed with the scalar product given by:

$$
<f_{1} \oplus \cdots \oplus f_{k}, h_{1} \oplus \cdots \oplus h_{k}>=\sum_{j=1}^{k}<f_{j}, h_{j}>
$$

where $\left\langle f_{j}, h_{j}\right\rangle=\int f_{j}(x) \overline{h_{j}(x)} d x$. We shall denote by $\pi_{j}: L^{2}\left(\mathbf{R}, \mathbf{C}^{n}\right) \rightarrow L^{2}(\mathbf{R})$ the canonical projection onto the jth component $1 \leq j \leq k: \pi_{j}\left(f_{1} \oplus \cdots \oplus f_{k}\right)=f_{j}$. For two vector parameters $\mathbf{a}=\left(a_{1}, \ldots, a_{k}\right) \in \overline{\mathbf{R}^{k}}, \mathbf{b}=\left(b_{1}, \ldots, b_{k}\right) \in \mathbf{R}^{k}$ we introduce the following unitary operators:

$$
\begin{gathered}
t_{\mathbf{a}, \mathbf{b}}: L^{2}\left(\mathbf{R}, \mathbf{C}^{n}\right) \rightarrow L^{2}\left(\mathbf{R}, \mathbf{C}^{n}\right), t_{\mathbf{a}, \mathbf{b}}=\oplus_{j=1}^{k} t_{a_{j}, b_{j}} \pi_{j} \\
w(\mathbf{a}, \mathbf{b}): L^{2}\left(\mathbf{R}, \mathbf{C}^{n}\right) \rightarrow L^{2}\left(\mathbf{R}, \mathbf{C}^{n}\right), w(\mathbf{a}, \mathbf{b})=\oplus_{j=1}^{k} w\left(a_{j}, b_{j}\right) \pi_{j}
\end{gathered}
$$

or, explicitly:

$$
\begin{gathered}
t_{\mathbf{a}, \mathbf{b}}\left(f_{1} \oplus \cdots \oplus f_{k}\right)=t_{a_{1}, b_{1}} f_{1} \oplus \cdots \oplus t_{a_{k}, b_{k}} f_{k} \\
w(\mathbf{a}, \mathbf{b})\left(f_{1} \oplus \cdots \oplus f_{k}\right)=w\left(a_{1}, b_{1}\right) f_{1} \oplus \cdots \oplus w\left(a_{k}, b_{k}\right) f_{k}
\end{gathered}
$$

Using the adjoints of each $t_{a_{j}, b_{j}}$ and $w\left(a_{j}, b_{j}\right)$ we get:

$$
\begin{gathered}
t_{\mathbf{a}, \mathbf{b}}^{*}=\oplus_{j=1}^{k} e^{-2 \pi i a_{j} b_{j}} t_{-a_{j,}-b_{j}} \pi_{j} \\
w(\mathbf{a}, \mathbf{b})^{*}=w(-\mathbf{a},-\mathbf{b})
\end{gathered}
$$

Consider now a vector $\mathbf{g}=g^{1} \oplus \cdots \oplus g^{k} \in L^{2}\left(\mathbf{R}, \mathbf{C}^{n}\right)$ and two positive vector parameters $\boldsymbol{\alpha}=\left(\alpha_{1}, \ldots, \alpha_{k}\right) \in \mathbf{R}_{+}^{k}$ and $\boldsymbol{\beta}=\left(\beta_{1}, \ldots, \beta_{k}\right) \in \mathbf{R}_{+}^{k}$. We construct two coherent sets using the previous unitary operators and the discrete lattice $\left\{(m \boldsymbol{\alpha}, n \boldsymbol{\beta}) ;(m, n) \in \mathbf{Z}^{2}\right\} \subset \mathbf{R}^{2 k}:$

$$
\begin{gathered}
\mathcal{G}_{\mathbf{g} ; \boldsymbol{\alpha}, \boldsymbol{\beta}}=\left\{t_{m \boldsymbol{\alpha}, n \boldsymbol{\beta}} \mathbf{g} ;(m, n) \in \mathbf{Z}^{2}\right\} \\
\mathcal{W}_{\mathbf{g} ; \boldsymbol{\alpha}, \boldsymbol{\beta}}=\left\{w(m \boldsymbol{\alpha}, n \boldsymbol{\beta}) \mathrm{g} ;(m, n) \in \mathbf{Z}^{2}\right\}
\end{gathered}
$$

Suppose either $\mathcal{G}_{\mathbf{g} ; \boldsymbol{\alpha}, \boldsymbol{\beta}}$ or $\mathcal{W}_{\mathbf{g} ; \boldsymbol{\alpha}, \boldsymbol{\beta}}$ is a frame in $L^{2}\left(\mathbf{R}, \mathbf{C}^{n}\right)$. We point out that, in general, one set is a frame does not imply that the other set is also a frame. Moreover, even if one set is a frame, the standard dual frame may not be a coherent frame (i.e. a frame of the same type). We shall derive conditions under which the standard dual frame is coherent. Before doing so we present an example of such multidimensional frame: 
Example 2.1. Consider $n=2, \alpha_{1}=\alpha_{2}=\frac{1}{2}, \beta_{1}=\beta_{2}=1$ and choose $g^{1}=$ $1_{[0,1]}, g^{2}=1_{[1,2]}$, the characteristic functions of, respectively, $[0,1]$ and $[1,2]$. We want to show that $\mathcal{G}_{g^{1} \oplus g^{2} ;\left(\frac{1}{2}, \frac{1}{2}\right),(1,1)}$ is a frame for $L^{2}(\mathbf{R}) \oplus L^{2}(\mathbf{R})$. A similar analysis works for $\mathcal{W}_{g^{1} \oplus g^{2} ;\left(\frac{1}{2}, \frac{1}{2}\right),(1,1)}$.

Consider two arbitrary functions $f_{1}, f_{2} \in \mathcal{S}$ in the space $\mathcal{S}$ of rapidly decaying functions. Then:

$$
\begin{aligned}
& c_{m n}^{1}=<f_{1}, g_{m n}^{1}>=\int_{n}^{n+1} e^{-i \pi m x} f_{1}(x) d x \\
& c_{m n}^{2}=<f_{2}, g_{m n}^{2}>=\int_{n+1}^{n+2} e^{-i \pi m x} f_{2}(x) d x
\end{aligned}
$$

Using the Poisson summation formula (see [Daub90]), $\sum_{m} e^{i \pi m x}=2 \sum_{m} \delta(x-$ $2 m$ ) we compute:

$$
\sum_{m, n} c_{m n}^{1} \overline{c_{m n}^{2}}=\sum_{n} \sum_{m} \int_{n}^{n+1} d x_{1} \int_{n+1}^{n+2} d x_{2} f_{1}\left(x_{1}\right) \overline{f_{2}\left(x_{2}\right)} e^{i \pi m\left(x_{2}-x_{1}\right)}=0
$$

Similarly, we get:

$$
\begin{gathered}
\sum_{m, n}\left|c_{m n}^{1}\right|^{2}=\sum_{n} \int_{n}^{n+1} d x_{1} \int_{n}^{n+1} d x_{2} f_{1}\left(x_{1}\right) \overline{f_{1}\left(x_{2}\right)} e^{i \pi m\left(x_{2}-x_{1}\right)}=2\left\|f_{1}\right\|^{2} \\
\sum_{m, n}\left|c_{m n}^{2}\right|^{2}=2\left\|f_{2}\right\|^{2}
\end{gathered}
$$

Hence we have:

$$
\sum_{m, n}\left|c_{m n}^{1}+c_{m n}^{2}\right|^{2}=2\left(\left\|f_{1}\right\|^{2}+\left\|f_{2}\right\|^{2}\right)
$$

and it follows that the frame operator on $\mathcal{S} \oplus \mathcal{S}$ is equal to $S=2 \cdot 1$. Since $\mathcal{S} \oplus \mathcal{S}$ is dense in $L^{2}(\mathbf{R}) \oplus L^{2}(\mathbf{R}), S=2 \cdot 1$ on the whole $L^{2}(\mathbf{R}) \oplus L^{2}(\mathbf{R})$. Thus $\mathcal{G}_{g^{1} \oplus g^{2} ;\left(\frac{1}{2}, \frac{1}{2}\right),(1,1)}$ is a tight frame in $L^{2}(\mathbf{R}) \oplus L^{2}(\mathbf{R})$. Moreover, as Theorem 2.6 will show, $\mathcal{G}_{g^{1} \oplus g^{2} ;\left(\frac{1}{2}, \frac{1}{2}\right),(1,1)}$ is also a Riesz basis for $L^{2}(\mathbf{R}) \oplus L^{2}(\mathbf{R})$. $\diamond$

Now, returning to the coherent frames (2.6) and (2.7), the frame operators are given by:

$$
\begin{gathered}
S^{\mathcal{G}}: L^{2}\left(\mathbf{R}, \mathbf{C}^{n}\right) \rightarrow L^{2}\left(\mathbf{R}, \mathbf{C}^{n}\right), S^{\mathcal{W}}: L^{2}\left(\mathbf{R}, \mathbf{C}^{n}\right) \rightarrow L^{2}\left(\mathbf{R}, \mathbf{C}^{n}\right) \\
S^{\mathcal{G}}(f)=\sum_{m, n}<f, t_{m \boldsymbol{\alpha}, n} \boldsymbol{\beta} \mathbf{g}>t_{m \boldsymbol{\alpha}, n \boldsymbol{\beta}} \mathbf{g} \\
S^{\mathcal{W}}(f)=\sum_{m, n}<f, w(m \boldsymbol{\alpha}, n \boldsymbol{\beta}) \mathbf{g}>w(m \boldsymbol{\alpha}, n \boldsymbol{\beta}) \mathbf{g}
\end{gathered}
$$

Thus the standard dual of $\mathcal{G}_{\mathrm{g} ; \boldsymbol{\alpha}, \boldsymbol{\beta}}$ is given by:

$$
\widetilde{\mathcal{G}} \widetilde{\mathbf{g}_{;} \boldsymbol{\alpha}, \boldsymbol{\beta}}=\left\{\left(S^{\mathcal{G}}\right)^{-1} t_{m \boldsymbol{\alpha}, n \boldsymbol{\beta}} \mathbf{g} ;(m, n) \in \mathbf{Z}^{2}\right\}
$$

and of $\mathcal{W}_{\mathbf{g} ; \boldsymbol{\alpha}, \boldsymbol{\beta}}$ by:

$$
\widetilde{\mathcal{W}_{\mathbf{g} ; \boldsymbol{\alpha}, \boldsymbol{\beta}}}=\left\{\left(S^{\mathcal{W}}\right)^{-1} w(m \boldsymbol{\alpha}, n \boldsymbol{\beta}) \mathrm{g} ;(m, n) \in \mathbf{Z}^{2}\right\}
$$

In order to state and prove our results, the following preliminary observations will be useful. Let us consider the sets $\mathcal{G}^{j}:=\mathcal{G}_{g^{j} ; \alpha_{j}, \beta_{j}}=\left\{t_{m \alpha_{j}, n \beta_{j}} g^{j} ;(m, n) \in \mathbf{Z}^{2}\right\}$ and $\mathcal{W}^{j}:=\mathcal{W}_{g^{j} ; \alpha_{j}, \beta_{j}}=\left\{w\left(m \alpha_{j}, n \beta_{j}\right) g^{j} ;(m, n) \in \mathbf{Z}^{2}\right\}$ for $1 \leq j \leq k$. They are the 
projections of $\mathcal{G}_{\mathbf{g} ; \boldsymbol{\alpha}, \boldsymbol{\beta}}$ and $\mathcal{W}_{\mathbf{g} ; \boldsymbol{\alpha}, \boldsymbol{\beta}}$ respectively, onto the components of $L^{2}\left(\mathbf{R}, \mathbf{C}^{n}\right)$ (i.e. $\left.\mathcal{G}^{j}=\pi_{j}\left(\mathcal{G}_{\mathbf{g} ; \boldsymbol{\alpha}, \boldsymbol{\beta}}\right) ; \mathcal{W}^{j}=\pi_{j}\left(\mathcal{W}_{\mathbf{g} ; \boldsymbol{\alpha}, \boldsymbol{\beta}}\right)\right)$. Then the following result holds.

Lemma 2.2. If $\mathcal{G}_{\mathbf{g} ; \boldsymbol{\alpha}, \boldsymbol{\beta}}$ is frame for $L^{2}\left(\mathbf{R}, \mathbf{C}^{n}\right)$ then each $\mathcal{G}^{j}$ is frame in $L^{2}(\mathbf{R})$, $1 \leq j \leq k$. If $\mathcal{W}_{\mathbf{g} ; \boldsymbol{\alpha}, \boldsymbol{\beta}}$ is frame for $L^{2}\left(\mathbf{R}, \mathbf{C}^{n}\right)$ then each $\mathcal{W}^{j}$ is frame in $L^{2}(\mathbf{R})$ However the converse is not true.

REMARK 2.3. Before proving this lemma we give an example where the converse is not true. Suppose $n=2$ and take $\alpha_{1}=\alpha_{2}, \beta_{1}=\beta_{2}$ and $g^{1}=g^{2}$ such that $\mathcal{G}_{g^{1} ; \alpha_{1}, \beta_{1}}$ be a frame in $L^{2}(\mathbf{R})$. Then $\mathcal{G}^{1}=\mathcal{G}^{2}$ and $\mathcal{W}^{1}=\mathcal{W}^{2}$ are all frames, but:

$$
\mathcal{G}_{\mathbf{g} ; \boldsymbol{\alpha}, \boldsymbol{\beta}}=\left\{g_{m n} \oplus g_{m n} ; g_{m n}=t_{m \alpha_{1}, n \beta_{1}} g^{1}, m, n \in \mathbf{Z}\right\}
$$

Thus the span of $\mathcal{G}_{\mathrm{g} ; \boldsymbol{\alpha}, \boldsymbol{\beta}}$ contains only vectors of the form $f \oplus f$, with $f \in L^{2}(\mathbf{R})$. Obviously $(-f) \oplus f$, for $f \neq 0$ is not in this span and therefore $\mathcal{G}_{\mathbf{g} ; \boldsymbol{\alpha}, \boldsymbol{\beta}}$ is not a frame in $L^{2}\left(\mathbf{R}, \mathbf{C}^{2}\right)$. Similarly for $\mathcal{W}_{\mathbf{g} ; \boldsymbol{\alpha}, \boldsymbol{\beta}}$.

Proof. The frame condition for $\mathcal{G}_{\mathbf{g} ; \boldsymbol{\alpha}, \boldsymbol{\beta}}$ reads as:

$$
A \sum_{j=1}^{k}\left\|f_{j}\right\|^{2} \leq \sum_{m, n}\left|\sum_{j=1}^{k}<f_{j}, t_{m \alpha_{j}, n \beta_{j}} g^{j}>\right|^{2} \leq B \sum_{j=1}^{k}\left\|f_{j}\right\|^{2}
$$

for any $f_{j} \in L^{2}(\mathbf{R})$. For $f_{j}=\delta_{j j_{0}} f$ we get:

$$
A\|f\|^{2} \leq \sum_{m, n}\left|<f, t_{m \alpha_{j_{0}}, n \beta_{j_{0}}} g^{j_{0}}>\right|^{2} \leq B\|f\|^{2}
$$

for any $f \in L^{2}(\mathbf{R})$ which means $\mathcal{G}^{j 0}$ is a frame for $L^{2}(\mathbf{R})$. A similar proof shows that each $\mathcal{W}^{j}$ is frame in $L^{2}(\mathbf{R})$ when $\mathcal{W}_{\mathbf{g} ; \boldsymbol{\alpha}, \boldsymbol{\beta}}$ is frame in $L^{2}\left(\mathbf{R}, \mathbf{C}^{n}\right)$.

We introduce now the notion of frame orthogonality:

Definition 2.4. Let $\mathcal{F}_{1}=\left\{g_{i}^{1} ; i \in \mathbf{I}\right\}$ and $\mathcal{F}_{2}=\left\{g_{i}^{2} ; i \in \mathbf{I}\right\}$ be two frames in some Hilbert space $K$. We say that $\mathcal{F}_{1}$ is orthogonal to $\mathcal{F}_{2}$ if for all $f, h \in K$ we have:

$$
\sum_{i \in \mathbf{I}}<f, g_{i}^{1}><g_{i}^{2}, h>=0
$$

Example 2.5. Consider the same example as before (Example 2.1). The equation (2.8) shows that condition (2.9) is fulfilled for any $f_{1}, f_{2} \in \mathcal{S}$. Since $\mathcal{S}$ is dense in $L^{2}(\mathbf{R})$ we get that $(2.8)$ holds for any $f_{1}, f_{2} \in L^{2}(\mathbf{R})$. $\diamond$

If we denote by $T_{1}: K \rightarrow l^{2}(\mathbf{I})$ and $T_{2}: K \rightarrow l^{2}(\mathbf{I})$ the analysis operators associated to $\mathcal{F}_{1}$ and $\mathcal{F}_{2}$, respectively, defined by $T_{1}(f)=\left\{<f, g_{i}^{1}>\right\}_{i \in \mathrm{I}}, T_{2}(f)=$ $\left.\left\{<f, g_{i}^{2}\right\rangle\right\}_{i \in \mathrm{I}}$, the condition (2.9) can be rewritten as $T_{1}^{*} T_{2}=0$.

Consider now the following three sets of conditions:

I. $\mathcal{G}^{j}$ is orthogonal to $\mathcal{G}^{l}$, for all $j \neq l, 1 \leq j, l \leq k$

II. $\mathcal{W}^{j}$ is orthogonal to $\mathcal{W}^{l}$, for all $j \neq l, 1 \leq j, l \leq k$

III. $\alpha_{1} \beta_{1}=\cdots=\alpha_{k} \beta_{k}=: \gamma$ ( $\gamma$ stands as a notation for the common value) 
THEOREM 2.6. With the notations introduced before:

a) If $\mathcal{G}_{\mathbf{g} ; \boldsymbol{\alpha}, \boldsymbol{\beta}}$ is a frame for $L^{2}\left(\mathbf{R}, \mathbf{C}^{n}\right)$ and I or III holds true, then its standard dual is also a Gabor frame generated by a vector $\mathbf{g}^{\mathcal{G}} \in L^{2}\left(\mathbf{R}, \mathbf{C}^{n}\right)$ (i.e. $\widetilde{\mathcal{G}_{\mathbf{g} ; \boldsymbol{\alpha}}, \boldsymbol{\beta}}=$ $\left.\mathcal{G}_{\mathbf{g}} \mathcal{G}_{\boldsymbol{\alpha}, \boldsymbol{\beta}}\right)$;

b) If $\mathcal{W}_{\mathbf{g} ; \boldsymbol{\alpha}, \boldsymbol{\beta}}$ is a frame for $L^{2}\left(\mathbf{R}, \mathbf{C}^{n}\right)$ and II or III holds true, then its standard dual is a also a Weyl-Heisenberg frame generated by a vector $\mathbf{g}^{\mathcal{W}} \in L^{2}\left(\mathbf{R}, \mathbf{C}^{n}\right)$ (i.e. $\left.\widetilde{\mathcal{W}_{\mathrm{g} ; \boldsymbol{\alpha}, \boldsymbol{\beta}}}=\mathcal{W}_{\mathrm{g}} \mathcal{W}_{; \boldsymbol{\alpha}, \boldsymbol{\beta}}\right)$

c) If III holds true then $\mathcal{G}_{\mathbf{g} ; \boldsymbol{\alpha}, \boldsymbol{\beta}}$ is a frame if and only if $\mathcal{W}_{\mathbf{g} ; \boldsymbol{\alpha}, \boldsymbol{\beta}}$ is a frame and in this case $\mathrm{g}^{\mathcal{G}}=\mathrm{g}^{\mathcal{W}}$;

d) If any of the above cases occurs then $\sum_{j=1}^{k} \alpha_{j} \beta_{j} \leq 1$;

e) Suppose $\mathcal{G}_{\mathbf{g}, \boldsymbol{\alpha}, \boldsymbol{\beta}}$ is a frame and I or III holds true. Then $\mathcal{G}_{\mathbf{g}, \boldsymbol{\alpha}, \boldsymbol{\beta}}$ is a Riesz basis for $L^{2}\left(\mathbf{R}, \mathbf{C}^{n}\right)$ if and only if $\sum_{j=1}^{k} \alpha_{j} \beta_{j}=1$;

f) Suppose $\mathcal{W}_{\mathbf{g} ; \boldsymbol{\alpha}, \boldsymbol{\beta}}$ is a frame and II or III holds true. Then $\mathcal{W}_{\mathbf{g} ; \boldsymbol{\alpha}, \boldsymbol{\beta}}$ is a Riesz basis for $L^{2}\left(\mathbf{R}, \mathbf{C}^{n}\right)$ if and only if $\sum_{j=1}^{k} \alpha_{j} \beta_{j}=1$.

Proof. a),b) In order to prove a), respectively b) it is enough to check that the corresponding frame operator commutes with $t_{m \boldsymbol{\alpha}, n \boldsymbol{\beta}}$, respectively $w(m \boldsymbol{\alpha}, n \boldsymbol{\beta})$. Consider the Gabor set.

If $\mathrm{I}$ is true then the frame operator decomposes into a diagonal sum of operators:

$$
S^{\mathcal{G}}=\oplus_{j=1}^{k} S^{j} \pi_{j}
$$

where $S^{j}=\sum_{m, n}<\cdot, t_{m \alpha_{j}, n \beta_{j}} g^{j}>t_{m \alpha_{j}, n \beta_{j}} g^{j}, 1 \leq j \leq k$.

Now, since $\left[S^{j}, t_{m \alpha_{j}, n \beta_{j}}\right]=0$ (see for instance [DLL95] relation (2.5)) we get that $\left[S^{\mathcal{G}},{ }_{m}{ }_{m, n} \boldsymbol{\beta}\right]=0$ for any $m, n \in \mathbf{Z}$, i.e. they commute (by $[\cdot, \cdot]$ we denote the commutator $[A, B]=A B-B A$ ).

If III is true we have:

$$
\begin{aligned}
& S^{\mathcal{G}} t_{m_{0} \boldsymbol{\alpha}, n_{0} \boldsymbol{\beta}} f=\sum_{m, n}<t_{m_{0} \boldsymbol{\alpha}_{, n_{0}} \boldsymbol{\beta}} f, t_{m \boldsymbol{\alpha}, n \boldsymbol{\beta}} \mathrm{g}>t_{m \boldsymbol{\alpha}, n \boldsymbol{\beta}} \mathrm{g} \\
& =\sum_{m, n}<f, e^{-2 \pi i m_{0} n_{0} \gamma} t_{-m_{0}} \boldsymbol{\alpha}_{i}-n_{0} \boldsymbol{\beta}^{t_{m} \boldsymbol{\alpha}, n \boldsymbol{\beta}} \mathrm{g}>t_{m \boldsymbol{\alpha}, n} \boldsymbol{\beta} \mathrm{g}
\end{aligned}
$$

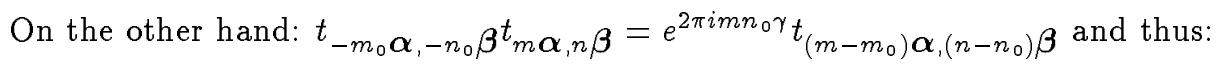

$$
\begin{aligned}
& S^{\mathcal{G}} t_{m_{0} \boldsymbol{\alpha}, n_{0} \boldsymbol{\beta}}=\sum_{m, n}<\cdot, e^{2 \pi i\left(m-m_{0}\right) n_{0} \gamma} t_{\left(m-m_{0}\right) \boldsymbol{\alpha},\left(n-n_{0}\right) \boldsymbol{\beta}} \mathbf{g}>t_{m \boldsymbol{\alpha}, n \boldsymbol{\beta}} \mathbf{g} \\
& =\sum_{m, n}^{m, n}<\cdot, t_{m \boldsymbol{\alpha}, n \boldsymbol{\beta}} \mathbf{g}>e^{-2 \pi i m n_{0} \gamma} t_{\left(m+m_{0}\right) \boldsymbol{\alpha},\left(n+n_{0}\right) \boldsymbol{\beta}} \mathbf{g} \\
& =\sum_{m, n}^{m, n}<\cdot, t_{m \boldsymbol{\alpha}, n \boldsymbol{\beta}} \mathbf{g}>t_{m_{0} \boldsymbol{\alpha}, n_{0}} \boldsymbol{\beta}_{m \boldsymbol{\alpha}, n \boldsymbol{\beta}} \mathbf{g}=t_{m_{0} \boldsymbol{\alpha}, n_{0} \boldsymbol{\beta}} S^{\mathcal{G}}
\end{aligned}
$$

For $S^{\mathcal{W}}$ the calculus goes in the same way but now: $w\left(-m_{0} \boldsymbol{\alpha},-n_{0} \boldsymbol{\beta}\right) w(m \boldsymbol{\alpha}, n \boldsymbol{\beta})=$ $e^{i \pi\left(m n_{0}-m_{0} n\right) \gamma} w\left(\left(m-m_{0}\right) \boldsymbol{\alpha},\left(n-n_{0}\right) \boldsymbol{\beta}\right)$.

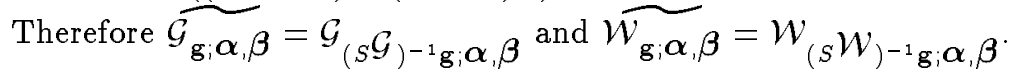

c) If III holds true we can check that $S^{\mathcal{G}}=S^{\mathcal{W}}$ and thus $\mathrm{g}^{\mathcal{G}}=\mathrm{g}^{\mathcal{W}}$. 
d),e),f) Since the frame operator commutes with $t_{m \boldsymbol{\alpha}, n \boldsymbol{\beta}}$, respectively $w(m \boldsymbol{\alpha}, n \boldsymbol{\beta})$ we get that the associated tight frame (defined by $\mathbf{g}_{m, n}^{\sharp}=S^{-1 / 2} \mathbf{g}_{m n}$ with $\mathbf{g}_{m n}$, respectively $S$ given by either $t_{m \boldsymbol{\alpha}, n} \boldsymbol{\beta}$, respectively $S^{\mathcal{G}}$ or $w(m \boldsymbol{\alpha}, n \boldsymbol{\beta}) \mathrm{g}$, respectively $\left.S^{\mathcal{W}}\right)$ is also coherent; moreover this tight frame $\mathcal{G}_{\mathbf{g} ; \boldsymbol{\alpha}, \boldsymbol{\beta}}^{\sharp}=\left\{t_{m \boldsymbol{\alpha}, n} \boldsymbol{\beta}^{\sharp} ;(m, n) \in\right.$ $\left.\mathbf{Z}^{2}\right\}$, or $\mathcal{W}_{\mathbf{g} ; \boldsymbol{\alpha}, \boldsymbol{\beta}}^{\sharp}=\left\{w(m \boldsymbol{\alpha}, n \boldsymbol{\beta}) \mathbf{g}^{\sharp} ;(m, n) \in \mathbf{Z}^{2}\right\}$, has frame bound 1. Then:

$$
\mathbf{f}=\sum_{m, n} \sum_{j=1}^{k}<\mathbf{f}, t_{m \boldsymbol{\alpha}, n} \boldsymbol{\beta} \mathbf{g}^{\sharp}>t_{m \boldsymbol{\alpha}, n} \boldsymbol{\beta} \mathbf{g}^{\sharp}, \forall \mathbf{f} \in L^{2}\left(\mathbf{R}, \mathbf{C}^{n}\right)
$$

which implies $\sum_{m, n}<f, t_{m \alpha_{j}, n \beta_{j}} g_{j}^{\sharp}>t_{m \alpha_{l}, n \beta_{l}} g_{l}^{\sharp}=\delta_{j l} f, \forall f \in L^{2}(\mathbf{R})$. Thus $\mathcal{G}_{j}^{\sharp}=\pi_{j}\left(\mathcal{G}_{\mathbf{g} ; \boldsymbol{\alpha}, \boldsymbol{\beta}}^{\sharp}\right)$ is a tight frame with bound 1 in $L^{2}(\mathbf{R})$ and from a necessary criterion (relation (2.2.9) in [Daub90]) we get $\left\|g_{j}^{\sharp}\right\|^{2}=\alpha_{j} \beta_{j}$.

On the one hand, from (2.10) for $\mathbf{f}=\mathrm{g}^{\natural}$ we get:

$$
\left\|\mathbf{g}^{\sharp}\right\|^{2}=\sum_{m, n} \sum_{j=1}^{k}\left|<g_{j}^{\sharp}, t_{m \alpha_{j}, n \beta_{j}} g_{j}^{\sharp}>\right|^{2} \geq\left\|\mathbf{g}^{\sharp}\right\|^{4}
$$

Thus $\left\|\mathbf{g}^{\sharp}\right\|^{2}=\sum_{j=1}^{k}\left\|g_{j}^{\sharp}\right\|^{2}=\sum_{j=1}^{k} \alpha_{j} \beta_{j} \leq 1$.

On the other hand, it is known that the frame is a Riesz basis if and only if the associated tight frame is an orthonormal basis. Thus $\left\|g^{\sharp}\right\|^{2}=1$ and the conclusion follows.

From this theorem one can see that the Gabor and Weyl-Heisenberg cases are very similar. However in the next section, where nonlocalization theorems are stated and proved, a difference emerges. We can handle the Weyl-Heisenberg case under the conditions II or III, but for the Gabor set we can treat only the case III.

\section{The Balian-Low type theorems for Riesz bases}

As we have proved in Theorem 2.6, if condition III holds true any result about Weyl-Heisenberg frames moves automatically into Gabor frames with the same lattice. We shall concentrate in this section on Weyl-Heisenberg Riesz bases. But before stating the results, we have to introduce some function spaces. Consider the following unbounded operators:

$$
q: L^{2}(\mathbf{R}) \rightarrow L^{2}(\mathbf{R}), D(q)=\left\{\left.f \in L^{2}(\mathbf{R})\left|\int\right| x f(x)\right|^{2} d x<\infty\right\}, q(f)(x)=x f(x)
$$

$$
p: L^{2}(\mathbf{R}) \rightarrow L^{2}(\mathbf{R}), D(p)=\left\{\left.f \in L^{2}(\mathbf{R})\left|\int\right| \frac{d f}{d x}\right|^{2} d x<\infty\right\}, p(f)(x)=i \frac{d f}{d x}
$$

where the derivative is considered in the distributional sense, and construct now similar operators on $L^{2}\left(\mathbf{R}, \mathbf{C}^{n}\right)$ :

$$
\begin{aligned}
& Q: L^{2}\left(\mathbf{R}, \mathbf{C}^{n}\right) \rightarrow L^{2}\left(\mathbf{R}, \mathbf{C}^{n}\right), D(Q)=\oplus_{j=1}^{k} D(q), Q=\oplus_{j=1}^{k} q \pi_{j} \\
& P: L^{2}\left(\mathbf{R}, \mathbf{C}^{n}\right) \rightarrow L^{2}\left(\mathbf{R}, \mathbf{C}^{n}\right), D(P)=\oplus_{j=1}^{k} D(p), P=\oplus_{j=1}^{k} p \pi_{j}
\end{aligned}
$$


Consider also the Wiener amalgam space (see [Feich90]):

$$
W\left(C_{0}, l^{1}\right)=\left\{f, f \text { continuous and }\|f\|_{W\left(L^{\infty}, l^{1}\right)}=\sum_{j}\left\|f \cdot 1_{[j, j+1]}\right\|_{\infty}<\infty\right\}
$$

a space of functions that will be useful in the third version of the BL theorem.

Now we state the "weak", "strong" and "amalgam" versions of the BL theorem for $L^{2}\left(\mathbf{R}, \mathbf{C}^{n}\right)$ (in the terminology of [BHW95]):

Lemma 3.1 (weak BLT for $L^{2}\left(\mathbf{R}, \mathbf{C}^{n}\right)$ ). Suppose $\mathrm{g} \in L^{2}\left(\mathbf{R}, \mathbf{C}^{n}\right)$ and $\boldsymbol{\alpha}, \boldsymbol{\beta} \in$ $\mathbf{R}_{+}^{k}$ such that II or III holds true and $\mathcal{W}_{\mathbf{g} ; \boldsymbol{\alpha}, \boldsymbol{\beta}}$ is a Riesz basis for $L^{2}\left(\mathbf{R}, \mathbf{C}^{n}\right)$. If $\tilde{\mathrm{g}}$ is the generator of the biorthogonal Riesz basis then either $\mathrm{g} \notin D(Q) \cap D(P)$ or $\tilde{\mathrm{g}} \notin D(Q) \cap D(P)$.

Theorem 3.2 (strong BLT for $L^{2}\left(\mathbf{R}, \mathrm{C}^{n}\right)$ ). Suppose $\mathrm{g} \in L^{2}\left(\mathbf{R}, \mathrm{C}^{n}\right)$ and $\boldsymbol{\alpha}, \boldsymbol{\beta} \in$ $\mathbf{R}_{+}^{k}$ such that II or III holds true and $\mathcal{W}_{\mathbf{g} ; \boldsymbol{\alpha}, \boldsymbol{\beta}}$ is a Riesz basis for $L^{2}\left(\mathbf{R}, \mathbf{C}^{n}\right)$. Then $\mathrm{g} \notin D(Q) \cap D(P)$.

REMARK 3.3. As stated here, Theorem 3.2 is clearly stronger than Lemma 3.1 . However, the technique (due to Battle) used in the proof of Lemma 3.1 also leads to a similar conclusion under slighty weaker conditions on $g$, when the hypotheses of Theorem 3.2 no longer hold true.

Theorem 3.4 (amalgam BLT for $L^{2}\left(\mathbf{R}, \mathbf{C}^{n}\right)$ ). Suppose $\mathbf{g} \in L^{2}\left(\mathbf{R}, \mathbf{C}^{n}\right)$ and $\boldsymbol{\alpha}, \boldsymbol{\beta} \in$ $\mathbf{R}_{+}^{k}$ such that III holds true and $\mathcal{W}_{\mathbf{g} ; \boldsymbol{\alpha}, \boldsymbol{\beta}}$ is a Riesz basis for $L^{2}\left(\mathbf{R}, \mathbf{C}^{n}\right)$. Then $\mathrm{g} \notin \oplus_{l=1}^{k} W\left(C_{0}, l^{1}\right)$ and $\hat{\mathrm{g}} \notin \oplus_{l=1}^{k} W\left(C_{0}, l^{1}\right)$ (where the hat ${ }^{\wedge}$ stands for the Fourier transform).

And now the proofs:

Proof of Lemma 3.1. The proof follows Battle's idea and is essentially similar to that given in his paper [Batt88] (see also [BHW95] or [DaJa93]).

For $\mathbf{a} \in \mathbf{R}^{k}$ and $\mathbf{g} \in L^{2}\left(\mathbf{R}, \mathbf{C}^{n}\right)$ we define $\mathbf{a g}=\left(a_{1} g_{1}, \ldots, a_{k} g_{k}\right)$, the componentwise multiplication. If $\mathbf{a} \in \mathbf{R}_{+}^{k}$ we denote $\mathbf{a}^{-1}=\left(a_{1}^{-1}, \ldots, a_{k}^{-1}\right)$.

The biorthogonality condition reads as:

$\sum_{m, n}<\cdot, w(m \boldsymbol{\alpha}, n \boldsymbol{\beta}) \mathbf{g}>w(m \boldsymbol{\alpha}, n \boldsymbol{\beta}) \tilde{\mathbf{g}}=\sum_{m, n}<\cdot, w(m \boldsymbol{\alpha}, n \boldsymbol{\beta}) \tilde{\mathbf{g}}>w(m \boldsymbol{\alpha}, n \boldsymbol{\beta}) \mathbf{g}=1_{L^{2}\left(\mathbf{R}_{,} \mathbf{C}^{n}\right)}$

Now suppose $\mathbf{g}, \tilde{\mathbf{g}} \in D(Q) \cap D(P)$. Then $w(m \boldsymbol{\alpha}, n \boldsymbol{\beta}) \mathbf{g}, w(m \boldsymbol{\alpha}, n \boldsymbol{\beta}) \tilde{\mathbf{g}} \in D(Q) \cap D(P)$ and:

$$
\begin{aligned}
& <P \boldsymbol{\alpha}^{-1} \mathbf{g}, Q \boldsymbol{\beta}^{-1} \tilde{\mathbf{g}}> \\
& =\sum_{m, n}<P \boldsymbol{\alpha}^{-1} \mathbf{g}, w(m \boldsymbol{\alpha}, n \boldsymbol{\beta}) \tilde{\mathbf{g}}><w(m \boldsymbol{\alpha}, n \boldsymbol{\beta}) \mathbf{g}, Q \boldsymbol{\beta}^{-1} \tilde{\mathbf{g}}> \\
& =\sum_{m, n}<\boldsymbol{\alpha}^{-1} \mathbf{g}, P w(m \boldsymbol{\alpha}, n \boldsymbol{\beta}) \tilde{\mathbf{g}}><Q w(m \boldsymbol{\alpha}, n \boldsymbol{\beta}) \mathbf{g}, \boldsymbol{\beta}^{-1} \tilde{\mathbf{g}}>
\end{aligned}
$$

On the other hand:

$$
P w(\mathbf{a}, \mathbf{b})=w(\mathbf{a}, \mathbf{b}) P-2 \pi \mathbf{a} w(\mathbf{a}, \mathbf{b}), Q w(\mathbf{a}, \mathbf{b})=w(\mathbf{a}, \mathbf{b}) Q+\mathbf{b} w(\mathbf{a}, \mathbf{b})
$$

Using biorthogonality:

$$
\begin{aligned}
<\boldsymbol{\alpha}^{-1} \mathrm{~g},-2 \pi m \boldsymbol{\alpha} w(m \boldsymbol{\alpha}, n \boldsymbol{\beta}) \tilde{\mathbf{g}}> & =-2 \pi m<\mathrm{g}, w(m \boldsymbol{\alpha}, n \boldsymbol{\beta}) \tilde{\mathrm{g}}> \\
= & -2 \pi m \delta_{m, 0} \delta_{n, 0}=0
\end{aligned}
$$


Similarly:

$$
<n \boldsymbol{\beta} w(m \boldsymbol{\alpha}, n \boldsymbol{\beta}) \mathrm{g}, \boldsymbol{\beta}^{-1} \tilde{\mathbf{g}}>=n<w(m \boldsymbol{\alpha}, n \boldsymbol{\beta}) \mathrm{g}, \tilde{\mathbf{g}}>=0
$$

Therefore:

$$
\begin{aligned}
& <P \boldsymbol{\alpha}^{-1} \mathbf{g}, Q \boldsymbol{\beta}^{-1} \tilde{\mathbf{g}}> \\
& =\sum_{m, n}<\boldsymbol{\alpha}^{-1} \mathbf{g}, w(m \boldsymbol{\alpha}, n \boldsymbol{\beta}) P \tilde{\mathbf{g}}><w(m \boldsymbol{\alpha}, n \boldsymbol{\beta}) Q \mathrm{~g}, \boldsymbol{\beta}^{-1} \tilde{\mathbf{g}}> \\
& =\sum_{m, n}<w(-m \boldsymbol{\alpha},-n \boldsymbol{\beta}) \boldsymbol{\alpha}^{-1} \mathbf{g}, P \tilde{\mathbf{g}}><Q \mathbf{g}, w(-m \boldsymbol{\alpha},-n \boldsymbol{\beta}) \boldsymbol{\beta}^{-1} \tilde{\mathbf{g}}>
\end{aligned}
$$

The following commutators are straightforward

$$
[w(\boldsymbol{\alpha}, \boldsymbol{\beta}), \mathbf{c}]=[P, \mathbf{c}]=[Q, \mathbf{c}]=0
$$

Therefore:

$$
\begin{aligned}
& <P \boldsymbol{\alpha}^{-1} \mathbf{g}, Q \boldsymbol{\beta}^{-1} \tilde{\mathbf{g}}> \\
& =\sum_{m, n}<\boldsymbol{\beta}^{-1} Q \mathbf{g}, w(m \boldsymbol{\alpha}, n \boldsymbol{\beta}) \tilde{\mathbf{g}}><w(m \boldsymbol{\alpha}, n \boldsymbol{\beta}) \mathrm{g}, \boldsymbol{\alpha}^{-1} P \tilde{\mathbf{g}}> \\
& =<\boldsymbol{\beta}^{-1} Q \mathbf{g}, \boldsymbol{\alpha}^{-1} P \tilde{\mathbf{g}}>=<Q \boldsymbol{\alpha}^{-1} \mathbf{g}, P \boldsymbol{\beta}^{-1} \tilde{\mathbf{g}}>
\end{aligned}
$$

Now, we can find sequences $\left(\mathbf{f}_{n}\right)_{n \in \mathrm{N}},\left(\mathbf{h}_{n}\right)_{n \in \mathrm{N}}$ in $\oplus_{j=1}^{k} C_{0}^{\infty}(\mathbf{R}) \subset D(P) \cap D(Q) \subset$ $L^{2}\left(\mathbf{R}, \mathbf{C}^{n}\right)$ such that $\left\|\mathbf{g}-\mathbf{f}_{n}\right\| \rightarrow 0,\left\|\tilde{\mathrm{g}}-\mathbf{h}_{n}\right\| \rightarrow 0,\left\|P \mathrm{~g}-P \mathbf{f}_{n}\right\| \rightarrow 0,\left\|P \tilde{\mathrm{g}}-P \mathbf{h}_{n}\right\| \rightarrow \mathbf{0}$, $\left\|Q \mathbf{g}-Q \mathbf{f}_{n}\right\| \rightarrow \mathbf{0},\left\|Q \tilde{g}-Q \mathbf{h}_{n}\right\| \rightarrow \mathbf{0}$. On the one hand:

$$
\begin{aligned}
& <P \boldsymbol{\alpha}^{-1} \mathbf{f}_{n}, Q \boldsymbol{\beta}^{-1} \mathbf{h}_{n}>-<Q \boldsymbol{\alpha}^{-1} \mathbf{f}_{n}, P \boldsymbol{\beta}^{-1} \mathbf{h}_{n}> \\
& =<[P, Q] \boldsymbol{\alpha}^{-1} \mathbf{f}_{n}, \boldsymbol{\beta}^{-1} \mathbf{h}_{n}>=i<\boldsymbol{\alpha}^{-1} \mathbf{f}_{n}, \boldsymbol{\beta}^{-1} \mathbf{h}_{n}>
\end{aligned}
$$

On the other hand, since the scalar product is continuous, we get by passing to limit and using (3.6):

$$
0=i\left\langle\boldsymbol{\alpha}^{-1} \mathbf{g}, \boldsymbol{\beta}^{-1} \tilde{\mathbf{g}}\right\rangle
$$

In case II, $\tilde{g}^{j}=\left(S^{j} \mathcal{W}\right)^{-1} g^{j}$ and therefore (3.8) implies:

$$
\sum_{j=1}^{k} \frac{1}{\alpha_{j} \beta_{j}}<g^{j},\left(S^{j} \mathcal{W}\right)^{-1} g^{j}>=0
$$

Since $\left(S^{\mathcal{W}}\right)^{-1}$ is a positive operator, each term is positive. Consequently each $g^{j}=0$. Contradiction!

In case III, $\boldsymbol{\alpha}^{-1} \boldsymbol{\beta}^{-1}=\frac{1}{\gamma} 1$ and thus (3.8) turns into:

$$
0=<\mathrm{g},\left(S^{\mathcal{W}}\right)^{-1} \mathrm{~g}>
$$

which again implies $\mathrm{g}=0$ and also a contradiction!

Proof of Theorem 3.2. The idea is to prove that $\mathrm{g} \in D(P) \cap D(Q)$ implies $\tilde{\mathrm{g}} \in D(P) \cap D(Q)$ and then the conclusion follows from lemma 3.1.

Firstly we consider the case II. Since $S^{\mathcal{W}}=\oplus_{j=1}^{k} S^{j} \pi_{j}$ we get that $\tilde{\mathrm{g}}=\oplus_{j=1}^{k} \tilde{g}^{j}$, i.e. the standard dual of $\mathcal{W}_{\mathrm{g} ; \boldsymbol{\alpha}, \boldsymbol{\beta}}$ is obtained as a direct sum of the standard duals of each component frame. Thus the problem reduces to a "scalar" WH frame: given $g \in L^{2}(\mathbf{R})$ and $\alpha, \beta>0$ prove that if $\mathcal{W}_{g ; \alpha, \beta}$ is a frame and $g \in D(p) \cap D(q)$ then the generator of the standard dual has the same smoothness and decay, i.e. $\tilde{g} \in D(p) \cap D(q)$. We prove one more ingredient for this, namely $\alpha \beta$ is rational. 
Indeed, suppose that not all $\gamma_{j}=\alpha_{j} \beta_{j}$ are rational. This together with $\sum_{j=1}^{k} \alpha_{j} \beta_{j}=1$ (since $\mathcal{W}_{\mathbf{g} ; \boldsymbol{\alpha}, \boldsymbol{\beta}}$ is a Riesz basis) would imply that there are two labels $j \neq l$ such that $\gamma_{j}-\gamma_{l}$ is irrational. From orthogonality we get:

$$
\sum_{m, n}<f^{\prime}, w\left(m \alpha_{j}, n \beta_{j}\right) g^{j}><w\left(m \alpha_{l}, n \beta_{l}\right) g^{l}, h^{\prime}>=0, \forall f^{\prime}, h^{\prime} \in L^{2}(\mathbf{R})
$$

For $f^{\prime}=w\left(m_{0} \alpha_{j}, n_{0} \beta_{j}\right) f$ and $h^{\prime}=w\left(m_{0} \alpha_{l}, n_{0} \beta_{l}\right) h$ we get:

$$
\sum_{m, n} e^{i \pi\left(m_{0} n-m n_{0}\right)\left(\gamma_{j}-\gamma_{l}\right)}<f, w\left(m \alpha_{j}, n \beta_{j}\right) g^{j}><w\left(m \alpha_{l}, n \beta_{l}\right) g^{l}, h>=0
$$

$\forall f, h \in L^{2}(\mathbf{R}), m_{0}, n_{0} \in \mathbf{Z}$. Let us denote by

$$
c_{n}=\sum_{m} e^{i \pi m n_{0}\left(\gamma_{j}-\gamma_{l}\right)}<f, w\left(m \alpha_{j}, n \beta_{j}\right) g^{j}><w\left(m \alpha_{l}, n \beta_{l}\right) g^{l}, h>
$$

It is easy to check that $c \in l^{1}(\mathbf{Z})$. Now consider the complex-valued function $t \mapsto F(t)=\sum_{n} e^{2 \pi i n t} c_{n}$. We know that $F\left(m_{0} \frac{\gamma_{j}-\gamma_{l}}{2}\right)=0, \forall m_{0} \in \mathbf{Z}$. Since $F$ is 1 -periodic and continuous and the set $\left\{m_{0} \frac{\gamma_{j}-\gamma_{l}}{2} \bmod 1 ; m_{0} \in \mathbf{Z}\right\}$ is dense in $[0,1]$ we get that $F \equiv 0$. Thus $c_{n}=0, \forall n$. Applying a similar argument, but now with $n_{0}$ as a free-parameter we obtain $<f, w\left(m \alpha_{j}, n \beta_{j}\right) g^{j}><w\left(m \alpha_{l}, n \beta_{l}\right) g^{l}, h>=0$ $\forall f, h \in L^{2}(\mathbf{R}), m, n \in \mathbf{Z}$ which means $\left\|g^{j}\right\| \cdot\left\|g^{l}\right\|=0$ and this is a contradiction with the assumption that $\mathcal{W}_{\mathbf{g} ; \boldsymbol{\alpha}, \boldsymbol{\beta}}$ is a frame in $L^{2}\left(\mathbf{R}, \mathrm{C}^{n}\right)$. Thus we proved that all $\gamma_{j}$ 's should be rational.

Now we come back to our problem: to prove that if $g \in D(p) \cap D(q)$ then $\tilde{g} \in D(p) \cap D(q)$ also. Suppose now that $\gamma=\alpha \beta=\frac{q}{p}$ for $p, q \in \mathbf{N},(p, q)=1$ (i.e. they are relatively prime). We shall use the Zak transform of $g$ defined as:

$$
G(t, s)=\frac{1}{\sqrt{\alpha}} \sum_{k \in \mathbf{Z}} e^{2 \pi i k t} g\left(\frac{s+k}{\alpha}\right), G \in L^{2}(\square)
$$

where $\square=[0,1] \times[0,1]$ (for more results about the Zak transform see [Daub90]). For the dual we shall denote by $\widetilde{G}$ the Zak transform of $\tilde{g}$. We also introduce the following notations:

$$
\begin{gathered}
\mathbf{G}(t, s)=\left[\begin{array}{c}
G(t, s) \\
G\left(t+\frac{1}{q}, s\right) \\
\vdots \\
G\left(t+\frac{q-1}{q}, s\right)
\end{array}\right], \tilde{\mathbf{G}}(t, s)=\left[\begin{array}{c}
\tilde{G}(t, s) \\
\tilde{G}\left(t+\frac{1}{q}, s\right) \\
\vdots \\
\tilde{G}\left(t+\frac{q-1}{q}, s\right)
\end{array}\right] \\
\mathbf{S}(t, s)=\sum_{j=0}^{p-1} \mathbf{G}\left(t, s+\frac{j q}{p}\right) \mathbf{G}^{T}\left(t, s+\frac{j q}{p}\right)
\end{gathered}
$$

Thus $\mathbf{G}(t, s)$ is a $q$-vector of functions whereas $\mathbf{S}(t, s)$ is a $q \times q$ matrix whose entries are:

$$
\mathbf{S}_{l r}(t, s)=\sum_{j=0}^{p-1} \overline{G\left(t+\frac{l-1}{q}, s+\frac{j q}{p}\right)} G\left(t+\frac{r-1}{q}, s+\frac{j q}{p}\right)
$$

It is known (see $\left[\mathbf{Z i Z e 9 3}\right.$ ) that $\widetilde{\mathbf{G}}=q \mathbf{S}^{-1} \mathbf{G}$ and the frame condition reduces to the operational condition $A \mathbf{I} \leq \mathbf{S}(t, s) \leq B \mathbf{I}$ for a.e. $(t, s) \in \square$. This implies 
$|G(t, s)| \leq \sqrt{\frac{B}{p}}$. Similarly, $B^{-1} \leq \mathbf{S}^{-1}=\sum_{j=0}^{p-1} \overline{\widetilde{\mathbf{G}}\left(\cdot, \cdot+\frac{j q}{p}\right)} \tilde{\mathbf{G}}^{T}\left(\cdot, \cdot+\frac{j q}{p}\right) \leq A^{-1}$ implies $|\widetilde{G}(t, s)| \leq \frac{1}{\sqrt{A p}}$ for a.e. $(t, s) \in \square$. The assertion $\tilde{g} \in D(p) \cap D(q)$ is equivalent to $\widetilde{G} \in W^{1,2}(\square)$ (see [DaJa93]) or $\widetilde{\mathbf{G}} \in W^{1,2}\left(\square, \mathbf{C}^{q}\right)$ where $W^{1,2}(\square)$ and $W^{1,2}\left(\square, \mathbf{C}^{q}\right)$ are given by:

$$
W^{1,2}(\square)=\left\{f: \square \rightarrow \mathrm{C}, \text { such that } f, \frac{D f}{D t}, \frac{D f}{D s} \in L^{2}(\square)\right\}
$$

$\left(\frac{D f}{D t}, \frac{D f}{D s}\right.$ are the derivatives of $f$ in distributional sense)

$$
W^{1,2}\left(\square, \mathbf{C}^{q}\right)=\left\{F: \square \rightarrow \mathbf{C}^{q}, \text { such that } F^{*} \cdot F \in W^{1,2}(\square)\right\}
$$

i.e. each component of the vector $F$ belongs to $W^{1,2}(\square)$.

Let $u$ stand for $t$ or $s$. Then:

$$
\frac{d}{d u} \tilde{\mathbf{G}}=-\mathbf{S}^{-1} \frac{d \mathbf{S}}{d u} \mathbf{S}^{-1} \mathbf{G}+\mathbf{S}^{-1} \frac{d \mathbf{G}}{d u}
$$

We need then to prove that each term belongs to $L^{2}\left(\square, \mathbf{C}^{q}\right)$. Since $\mathbf{G} \in W^{1,2}\left(\square ; \mathbf{C}^{q}\right)$ and $\mathbf{S}^{-1} \leq A^{-1}$ we have $\mathbf{S}^{-1} \frac{d \mathbf{G}}{d u} \in L^{2}\left(\square ; \mathbf{C}^{q}\right)$. For the first term we need to prove that $\frac{d \mathbf{S}}{d u} \tilde{\mathbf{G}} \in L^{2}\left(\square ; \mathbf{C}^{q}\right)$. But the entries in this vector are sums of terms of the following form $\frac{d \bar{G}}{d u}\left(\cdot+x_{1}, \cdot+y_{1}\right) G\left(\cdot+x_{2}, \cdot+y_{2}\right) \tilde{G}\left(\cdot+x_{3}, \cdot+y_{3}\right)$ which clearly belong to $L^{2}(\square)$. This ends the proof of this case.

Finally, let us consider the case III. Since $\mathcal{W}_{\mathbf{g} ; \boldsymbol{\alpha}, \boldsymbol{\beta}}$ is a Riesz basis, we get $\gamma=\frac{1}{k}$. We scale the generators to obtain $\alpha_{1}=\cdots=\alpha_{k}$ and $\beta_{1}=\cdots=\beta_{k}$.

After some computation, one can check formally that:

$$
\sum_{m, n}<\mathbf{f}, w(m \boldsymbol{\alpha}, n \boldsymbol{\beta}) \mathbf{v}><w(m \boldsymbol{\alpha}, n \boldsymbol{\beta}) \mathbf{w}, \mathbf{h}>=\iint_{\square} d s d t \mathbf{F}^{T} \mathbf{M} \overline{\mathbf{H}}
$$

with:

$$
\mathbf{F}=\left[\begin{array}{c}
F_{1} \\
\vdots \\
F_{k}
\end{array}\right], \mathbf{H}=\left[\begin{array}{c}
H_{1} \\
\vdots \\
H_{k}
\end{array}\right], \mathbf{M}_{j l}(t, s)=\sum_{r=0}^{k-1} \overline{V_{j}\left(t, s+\frac{r}{k}\right)} W_{l}\left(t, s+\frac{r}{k}\right)
$$

where $F_{j}, H_{j}, V_{j}, W_{j}$ are the Zak transforms of $f_{j}, h_{j}, v_{j}$ and respectively $w_{j}$.

Let $\mathbf{S}: \square \rightarrow \mathbf{C}^{k \times k}$ be the $k \times k$ matrix $\mathbf{S}_{j l}(t, s)=\sum_{r=0}^{k-1} \overline{G_{j}\left(t, s+\frac{r}{k}\right)} G_{l}\left(t, s+\frac{r}{k}\right)$ or, more compactly

$$
\mathbf{S}=\sum_{r=0}^{k-1} \overline{\mathbf{G}\left(\cdot, \cdot+\frac{r}{k}\right)} \mathbf{G}^{T}\left(\cdot, \cdot+\frac{r}{k}\right), \text { where } \mathbf{G}(t, s)=\left[\begin{array}{c}
G_{1}(t, s) \\
\vdots \\
G_{k}(t, s)
\end{array}\right]
$$

The frame condition for $\mathcal{W}_{\mathbf{g} ; \boldsymbol{\alpha}, \boldsymbol{\beta}}$ reduces to $0<A \leq \mathbf{S}(t, s) \leq B<\infty$, a.e. $(t, s) \in \square$. Then the dual is given by $\widetilde{\mathbf{G}}=\mathbf{S}^{-1} \mathbf{G}$ and the proof is similar to the previous case: from $\mathbf{G} \in W^{1,2}\left(\square ; \mathbf{C}^{k}\right)$ it follows that $\tilde{\mathbf{G}} \in W^{1,2}\left(\square ; \mathbf{C}^{k}\right)$ which is equivalent to $\tilde{\mathrm{g}} \in D(P) \cap D(Q)$.

Proof of Theorem 3.4. Firstly, as before we can assume $\alpha_{j}=1$ and $\beta_{j}=\frac{1}{k}$ (otherwise we scale all generators $g^{j}$ ). Then, as we have shown before, the Riesz basis condition is equivalent to $A \leq \mathbf{S}(t, s) \leq B$, a.e. $(t, s) \in \square$. Let $T: \square \rightarrow \mathbf{C}^{k \times k}$ be the matrix whose entries are $T_{j l}(t, s)=\bar{G}_{j}\left(t, s+\frac{l-1}{k}\right)$. Then $\mathbf{S}=\bar{T} T^{T}$ and thus $\operatorname{det} \mathbf{S}=|\operatorname{det} T|^{2} \geq A$, a.e. $(t, s) \in \square$. Suppose $\mathbf{g} \in \oplus_{l=1}^{k} W\left(C_{0}, l^{1}\right)$. This implies 
that each $G_{j}(t, s)$ is continuous (see [BHW95]). Thus $\operatorname{det} T: \square \rightarrow \mathbf{C}$ is continuous and since $|\operatorname{det} T| \geq \sqrt{A}>0, \forall(t, s) \in \square$ we can define a continuous function $\varphi: \square \rightarrow \mathrm{C}$ such that $\operatorname{det} T(t, s)=|\operatorname{det} T(t, s)| e^{i \varphi(t, s)}$. One can easily check that $\operatorname{det} T(t+1, s)=\operatorname{det} T(t, s)$ and $\operatorname{det} T\left(t, s+\frac{1}{k}\right)=e^{-2 \pi i t}(-1)^{k-1} \operatorname{det} T(t, s)$. Therefore there are integers $M, N \in \mathbf{Z}$ such that:

$$
\varphi(t+1, s)=\varphi(t, s)+2 \pi M, \varphi\left(t, s+\frac{1}{k}\right)=\varphi(t, s)-2 \pi t+\pi(k-1)+2 \pi N
$$

Then:

$$
\begin{gathered}
0=\left(\varphi(0,0)-\varphi\left(0, \frac{1}{k}\right)\right)+\left(\varphi\left(0, \frac{1}{k}\right)-\varphi\left(1, \frac{1}{k}\right)\right)+\left(\varphi\left(1, \frac{1}{k}\right)-\varphi(1,0)\right)+(\varphi(1,0)-\varphi(0,0))= \\
=-\pi(k-1)-2 \pi N-2 \pi M-2 \pi+\pi(k-1)+2 \pi N+2 \pi M=-2 \pi
\end{gathered}
$$

Contradiction! Therefore $\mathrm{g} \notin \oplus_{j=1}^{k} W\left(C_{0}, l^{1}\right)$. Similarly, if we use the Zak transform of the Fourier transform of $g^{j}$ 's we get a similar conclusion for $\hat{\mathrm{g}}$.

\section{Conclusions}

In this paper we study some extensions of Gabor and Weyl-Heisenberg frames from unisignal systems $\left(L^{2}(\mathbf{R})\right)$ to multisignal systems $\left(L^{2}(\mathbf{R}) \oplus \cdots \oplus L^{2}(\mathbf{R})\right)$. For certain categories of such frames we proved that the standard dual is coherent. In some cases we have obtained extensions of the Balian-Low theorem in all three forms (weak, strong and amalgam). In future works we shall study the geometry of multisignal systems as well as some adjoint Riesz bases that can naturally be associated to such frames.

\section{References}

[Bali81] R.Balian, Un principe d'incertitude fort en théorie du signal on en mécanique quantique, C.R.Acad.Sci. Paris, 292 (1981), 1357-1362

[Batt88] G.Battle, Heisenberg Proof of the Balian-Low Theorem, Letters in Mathematical Physics, 15 (1988), 175-177

[BHW95] J.J.Benedetto, C.Heil, D.F.Walnut, Differentiation and the Balian-Low Theorem, J.Fourier Anal.Applic., vol.1, no.4 (1995), 355-402

[Daub90] I.Daubechies, The Wavelet Transform, Time-Frequency Localization and Signal Analysis, IEEE Trans.Inform. Theory, no.5, 36 (1990), 961-1005

[DaJa93] I.Daubechies, A.J.E.M.Janssen, Two Theorems on Lattice Expansions, IEEE Trans.on Info.Th., vol.39, no.1 (1993), 3-6

[DLL95] I.Daubechies, H.J.Landau, Z.Landau, Gabor Time-Frequency Lattices and the WexlerRaz Identity, J.Fourier Anal.Applic., vol.1, no.4 (1995), 437-478

[Feich90] H.Feichtinger, Generalized amalgams, with applications to Fourier transform, Canad.J.Math. 19, (1990), 394-409

[HeWa89] C.Heil, D.Walnut, Continuous and Discrete Wavelet Transforms, SIAM Review 31, no.4 (1989), 628-666

[Low85] F.Low, Complete sets of wave packets, A Passion for Physics - Essays in Honor of Geoffrey Chew, World Scientific, Singapore, 1985, 17-22

[You80] R.M.Young, An Introduction to Nonharmonic Fourier Series, Academic Press, 1980

[ZiZe93] M.Zibulski, Y.Y.Zeevi, Oversampling in the Gabor Scheme, IEEE Trans.on Sig.Proc., vol.41, no.8 (1996), 2679-2687

[ZiZe95] M.Zibulski, Y.Y.Zeevi, Analysis of Multi-Window Gabor-Type Schemes by Frame Methods, CC PUB \# 101, Israel Institute of Technology, April 1995

Program in Applied and Computational Mathematics, Princeton University, PrinceTON, NJ 08544

E-mail address: rvbalan@math.princeton.edu 